\title{
Use of a surface-mounted chirped fibre Bragg grating sensor to monitor delamination growth in a double-cantilever beam test
}

\section{A.R.Sanderson ${ }^{a^{*}}$, S.L.Ogin ${ }^{a}$, A.D.Crocombe ${ }^{a}$, M.R.L Gower ${ }^{b}$, R.J. Lee}

${ }^{a}$ Faculty of Engineering and Physical Sciences, University of Surrey, Guildford, GU2 7XH, United Kingdom. Tel.: 07769687703. E-mail address:

\author{
A.R.Sanderson@surrey.ac.uk \\ bational Physical Laboratory, Hampton Road, Teddington, Middlesex, TW11 0LW, \\ United Kingdom. \\ ${ }^{c}$ ESR Technology Ltd, 16 North Central 127, Milton Park, Abingdon, Oxfordshire \\ OX14 4SA, United Kingdom.
}

\begin{abstract}
A surface-mounted chirped fibre Bragg grating (CFBG) sensor has been used for the first time to monitor delamination growth within a composite material (a transparent, unidirectionally reinforced glass fibre/epoxy resin double-cantilever beam (DCB) specimen). The specimens were tested using a constant displacement rate, with the delamination length being measured using complementary techniques: (i) in situ photography, (ii) surface-mounted strain gauges, and (iii) the surface-mounted CFBG sensors. The unidirectionally reinforced DCB specimens showed characteristics typical of such material which complicate the curvature of the beams, i.e. the development of extensive fibre bridging and pronounced R-curve behaviour. To validate the interpretation of the CFBG reflected spectrum, the experimentally determined strains
\end{abstract}


from the surface-mounted strain gauges have been used, together with in situ photographs of the position of the delamination front. Using the CFBG sensor technique, the delamination length was measured to within about $4 \mathrm{~mm}$ over the $60 \mathrm{~mm}$ sensor length.

Keywords: A. Polymer-matrix composite (PMCs); A. Smart materials; B. Delamination; C. Fibre bridging; Chirped fibre Bragg grating (CFBG)

\section{Introduction}

Monitoring delamination growth in composite structures is an area of increasing interest, particularly with the expanding use of large composite structures, such as wind turbine blades. There is a need both to be able to determine whether a delamination has initiated, and also to determine the current location of the damage, and/or its growth rate. Various NDE (non-destructive evaluation) techniques are potentially able to detect the location of delaminations, including acoustic emission, electric resistance measurements, shearography and thermography [1-4]. However, it is likely to be difficult to employ many of the currently proposed techniques either due to the unproven nature of the technique or the physical difficulties of implementation (e.g. complications arising out of the connections required). With regard to fibre optic sensors, both the ability to be able to embed such sensors within a composite structure and the proven usage of fibre optical sensors for monitoring large structures [e.g. 5] make these sensors important candidates for monitoring delaminations.

Chirped FBG (CFBG) sensors embedded within composite materials have been shown to be able to monitor delamination growth in single-lap joints [6-8], where the 
changes in the reflected spectra due to delamination growth are much easier to interpret than for uniform FBGs. Whereas unstrained uniform FBG sensors have a constant periodic variation of the refractive index within the fibre core (the "grating" spacing), the CFBGs used in this work have a linearly increasing grating spacing. The relative ease of interpretation of CFBG reflected spectra with regard to damage development is a consequence of the relationship between the spectral bandwidth of the reflected spectrum (typically $20 \mathrm{~nm}$ ) and the sensor length (typically $60 \mathrm{~mm}$ ). If the strain field is perturbed by damage in the composite, so that the smooth linear increase in the grating spacing is disrupted, then a perturbation appears in the reflected spectrum that can be used to monitor the physical location of the damage. To monitor the propagation of damage in the form of delaminations in a single-lap joint (due to fatigue loading, for example), examination of successive spectra enables both the direction of growth of the delamination to be determined, as well as the current position of the delamination front to within a few millimetres. This is because the strain distribution associated with the delamination produces a well-defined perturbation in the reflected spectra (generally, either a peak or a trough in the spectrum). In order to determine the location of the disbond front more precisely, it is necessary to predict the spectra. In general, this requires that the strain distribution associated with the damage is ascertained, either using either finite-element analysis [e.g. 6], or through a closed form solution [e.g. 7], if available. In either case, the purpose of the analysis is to determine the strain distribution associated with a known location of the delamination front in relation to the sensor, in order to be able to predict the spectrum. Of course, if an alternative means of evaluating the strain distribution is available (as in the experiments to be described here), then a numerical analysis is not necessary. 
In many situations, it will be impractical to embed CFBG sensors within the composite structure under investigation (for example, a plate-bonded repair to a bridge beam) and hence it is important to determine whether a surface-bonded sensor can also monitor delamination growth. Consequently, in the current paper, the previous use of embedded CFBG sensors to monitor the progression of a delamination has been extended to the use of surface-bonded sensors.

\section{Experimental methods}

\subsection{Materials}

The material used in this study was a transparent, unidirectional GFRP laminate manufactured using a frame-winding technique, described in detail elsewhere [e.g. 9], consisting of E-glass fibres $(\mathrm{Vf} \approx 0.6)$ in a Shell Epikote 828 epoxy resin matrix. The specimens had nominal dimensions of $150 \mathrm{~mm}$ x $25 \mathrm{~mm}$ x $5 \mathrm{~mm}$, and a $40 \mathrm{~mm}$ long nylon film (with a thickness of $50 \mu \mathrm{m}$ ) to initiate the delamination, spanning the entire width of the specimen at the centre line (see figure 1). On both the upper and lower surfaces of the specimen at one end, aluminium brackets were bonded using DP-490 adhesive to enable the specimen to be loaded in the testing machine. A $60 \mathrm{~mm}$ section of each specimen was instrumented on one surface, beyond the position of the end of the $40 \mathrm{~mm}$ nylon insert, with strain gauges and a CFBG sensor. At the centre-line of one half of the DCB specimens, six $1 \mathrm{~mm}$ gauge length strain gauges (type: N11-FA-1-12023) were bonded with a nominal spacing of $10 \mathrm{~mm}$ apart, with the first gauge located 5 $\mathrm{mm}$ from the end of the insert. On the adjacent half-strip of the specimens, a CFBG sensor (manufactured by TeraXion) was bonded to the surface of the specimen, parallel 
to the length of the specimen and the strain gauges, using M-Bond 610 adhesive. The CFBG sensor had a $60 \mathrm{~mm}$ gauge length, a full width at the half-maximum of the reflected spectrum of $20 \mathrm{~nm}$, and a centre wavelength of $1550 \mathrm{~nm}$.

The strain gauges were monitored using a multi-channel data logger (Solitron Orion 3503) which enabled the six strain gauges to be logged simultaneously. The optical fibre sensor was connected to a Smart Fibres W-4 laser interrogator [10].

\subsection{Method}

The DCB specimens were loaded quasi-statically using a computer-controlled servohydraulic testing machine (Instron 4505) at a constant displacement rate of 1 $\mathrm{mm} / \mathrm{min}$. A USB camera was used to photograph the specimens in transmitted light every 60 seconds, with the specimens viewed from the face opposite to the instrumented face. The strain gauge data and the CFBG spectra were logged automatically every 2 seconds and 60 seconds, respectively. The test was stopped after the delamination had propagated beyond the $60 \mathrm{~mm}$ sensor region, at which point the opening displacement at the loading points had reached about $35 \mathrm{~mm}$.

\section{Results and discussion}

\subsection{Introduction}

In this section, the general behaviour of the DCB specimens is described in the context of the photographic measurements of delamination growth, the observation of the expected R-curve behaviour of the material (shown by the interlaminar toughness), and 
the relationship between the growth of the delamination and the measurements from the surface-mounted strain gauges. This is followed by a description of the changes in the reflected spectra recorded by the surface-mounted CFBG sensors for increasing delamination lengths.

\subsection{Delamination length measurements, interlaminar toughness and surface strain} measurements

During testing, the delamination initiated from the location of the nylon film insert and propagated along the specimen with increasing displacement of the DCB arms (note that all delamination lengths quoted below are measured from the end of the nylon insert, so that $40 \mathrm{~mm}$ needs to be added to these values to obtain the total delamination length). Due to the transparency of the specimens, the delamination front position can be determined easily from the in situ photographs. Figure 2, for example, shows a comparison of photographs of the $60 \mathrm{~mm}$ instrumented portion of the specimen for different delamination lengths. The delamination lengths shown here are measured along the centre-line of the specimen; it is clear that there is a slight curvature of the delamination front, so that the delamination lengths that would be measured at the edge of the specimens would be up to approximately $2.5 \mathrm{~mm}$ shorter in length.

To confirm that the position of the delamination front obtained from these in situ photographs provides an accurate location of the actual position of the delamination at the specimen mid-plane, a number of specimens were sectioned and polished in crosssection. The delamination front position as seen on the upper and lower surface of the specimen was marked, and a cross-section cut and polished, parallel to the length of the specimen, at that position. Microscopical examination of the cross-sections showed that 
the plan-view indication of the position of the delamination front was accurate to within about $0.25 \mathrm{~mm}$ of the actual delamination position at the specimen mid-plane.

The load-displacement curves for the DCB tests enabled the toughness, $G_{c}$, to be determined using the well-known expression

$$
G_{c}=\frac{n P u}{2 t a}
$$

Equation 1

In equation $1, P$ is the load for a given crosshead displacement, $u$, the thickness of the specimen is $t$, and $a$ is the delamination length. $n$ is the value of the exponent in the relationship between the specimen compliance, $C$, and the delamination length (i.e. $C=$ $k a^{n}$, where $k$ is a constant); the value of $n$ is 3 for these unidirectionally reinforced GFRP specimens [11]. Figure 3 shows a typical plot of toughness against delamination length; the R-curve behaviour (with a delamination initiation value of about $200 \mathrm{~J} / \mathrm{m}^{2}$, and plateau value of about $800 \mathrm{~J} / \mathrm{m}^{2}$ ), is typical of these specimens [11]. As is wellknown, the development of the R-curve for such materials is related to fibre bridging, and a typical example of fibre bridging in these specimens can be seen in figure 4 . In this work, the development of fibre bridging is important since, as will be seen below, the crack closing forces associated with the fibre bridging complicate the curvature of the arms of the DCB specimen. The R-curve shows that the fibre bridging is not fully developed until delamination lengths of about $35 \mathrm{~mm}$ have been reached, and this is reflected in the measurements of the surface strains recorded by the surface-mounted strain gauges.

Figure 5 shows an example of the strain changes measured by each of the six strain gauges as a function of the delamination length where strain gauge 1 (SG1) is the strain gauge closest to the insert; the centre of SG1 is located $5 \mathrm{~mm}$ from the end of the 
insert and, of course, the delamination passes adjacent to this strain gauge first. Six vertical lines have been included in Figure 5, showing the actual position of the centre of each strain gauge with respect to the delamination length (nominally, the gauges were $10 \mathrm{~mm}$ apart). The strain gauges were bonded to the surface of the DCB specimen so that the strains recorded are all compressive.

The strain gauge results from these experiments are similar to the strain data derived by Stutz et al [12] from their multiplexed FBGs positioned close to the midplane of a unidirectionally reinforced CFRP laminate tested as a DCB specimen. The following observations can be made. First, the strain gauges detect the effect of the delamination when the delamination is approximately $10-15 \mathrm{~mm}$ from the position of the strain gauge (Stutz et al [12] found this distance to be about $18 \mathrm{~mm}$ in their specimens). For example, SG2 detects an increasingly negative strain when the delamination is about $11 \mathrm{~mm}$ from the centre of the strain gauge. As the delamination approaches the position of the strain gauge, the compressive strain measured by the strain gauge increases rapidly and there is an observable change in the slope of the strain/delamination length curve when the delamination has grown to a position immediately adjacent to the strain gauge, due to the separation and bending of the DCB arms. The radius of curvature of the DCB arm when the delamination front passes adjacent to each strain gauge initially decreases with delamination length (as indicated by the increasing compressive strain recorded by SG1, SG2 and SG3) and then reaches an approximately constant value for delamination lengths greater than about $30 \mathrm{~mm}$ (for SG4, SG5 and SG6). This is a consequence of the full development of fibre bridging when the delamination has reached a length of about 30 to $35 \mathrm{~mm}$, and also corresponds to the length of the delamination for the development of the plateau in the R-curve (see figure 3). The peak in the compressive strain for each strain gauge occurs when the 
delamination has passed about $10-15 \mathrm{~mm}$ beyond the strain gauge, since in order to overcome the effect of fibre bridging, additional bending (and hence, curvature) of the DCB arms is required. Finally, once the delamination front has moved well beyond each strain gauge (i.e. by a distance of about $10-15 \mathrm{~mm}$ ), the compressive strains recorded by the gauges reduce as the radius of curvature of the DCB arms relaxes.

The changes recorded in the strains measured by the surface-bonded strain gauges as the delamination grows indicate the complexity of the deformation of the DCB arms in the vicinity of the delamination front due to the development of fibre bridging. In this work, this complexity is important in the context of predicting the CFBG sensor results which are described in the next section.

\subsection{CFBG sensor results for the DCB tests}

Typical changes in the reflected spectra for a surface-bonded CFBG sensor for increasing delamination lengths are shown in figure 6 . The sensor was oriented so that the low wavelength end of the sensor was near to the nylon insert (again, all delamination lengths quoted in this section are additional to the $40 \mathrm{~mm}$ insert length).

Figure 6(a) shows the reflected spectra before delamination growth (i.e. for 0 $\mathrm{mm}$ ), and after the delamination has grown $20 \mathrm{~mm}$. There are two noticeable changes in the reflected spectra as a consequence of delamination growth. The first is the shift of the low wavelength end of the reflected spectrum to lower wavelengths, as indicated by the arrow in the figure. This can be understood by considering the grating spacings for the portion of the CFBG sensor attached to that part of the DCB arm that has currently delaminated; the compressive surface strains due to the curvature of the DCB arm reduce the grating spacings and hence shift the lower wavelength end of the spectrum to 
lower wavelengths. The second feature is the development of a perturbation in the spectrum (i.e. an extended dip in intensity) with a minimum at approximately $1543 \mathrm{~nm}$. This can be understood qualitatively with reference to changes in the grating spacings in the light of the strain gauge results described in Section 3.2. The intensity at any given wavelength is a function of the number of gratings with a spacing which corresponds, approximately, to that particular wavelength. The strain gauge results of Section 3.2 have shown that the compressive surface strain for distances greater than about 10-15 $\mathrm{mm}$ ahead of the delamination is negligible. Consequently, for the $20 \mathrm{~mm}$ long delamination of figure $6(\mathrm{a})$, the grating spacings for the $25-30 \mathrm{~mm}$ portion of the sensor nearest to the high-wavelength end are currently undisturbed. However, there is a rapidly increasing compressive surface strain as the position of the delamination front is approached, which causes the grating spacings to be increasingly compressed up to the position of the delamination front; behind the delamination front, although the compressive strains remain high, the strain gradient decreases. For CFBG sensors, it is high strain gradients which cause significant perturbations in the reflected spectra (uniform strains just shift the spectra to higher or lower wavelengths, depending on the sign of the strain). Consequently, the dip in intensity corresponds with the approximate location of the delamination front, and the location and direction of the delamination front can be tracked by monitoring the movement of the perturbation.

As figures 6(b) and 6(c) show, the perturbation in the spectrum moves to higher wavelengths as the delamination grows, since the high strain gradients are associated with the current position of the delamination front. Additionally, as the delamination front progresses to greater distances from the physical location of the low-wavelength end of the CFBG sensor, the low-wavelength end of the spectrum moves to slightly higher reflected wavelengths because the curvature of the DCB arm relaxes. Similarly, 
as the delamination front nears the position of the high- wavelength end of the sensor, such as with the delamination length of $57.1 \mathrm{~mm}$ (figure 6(c)), the spectrum shows the movement of the high-wavelength end of the reflected spectrum to lower wavelengths due to the compressive strains associated with the curvature of the DCB arm.

In summary, the perturbation in the reflected spectrum is a measure of the current location of the delamination. However, in order to be able to identify the location of the delamination front more precisely within the reflected spectrum, it is important to be able to predict the spectrum, and to locate the delamination front position within the spectrum.

\section{Modelling and prediction of the reflected spectrum of the CFBG sensors}

Predictions of changes to the reflected spectra of CFBG sensors can be made using a commercially available programme (OptiGrating; [13]) once the local strain distribution is known. In the present work, either a finite-element analysis of the DCB can be used, or the surface strain measurements provided by the strain gauges can be used (such strain gauge results would not be available for real applications, of course).

The surface strain distribution obtained from a 2D finite-element model of the DCB is shown in figure 7. As the fibre was positioned at the centre of the DCB (in the width-wise direction), plane strain (4 or 8 noded) elements were used; the experimentally determined properties of the UD GFRP material used in the model are shown in Table 1. The DCB was modelled for a delamination length of $34 \mathrm{~mm}$ (i.e. a total delamination length of $74 \mathrm{~mm}$ ) with a range of different mesh sizes, with the finest mesh size $(0.0024 \mathrm{~mm} \times 0.0024 \mathrm{~mm})$ at the location of the delamination front. This mesh size was significantly smaller than that required to provide convergence of the 
results. Figure 7 shows the predicted surface strain distribution approximately $30 \mathrm{~mm}$ either side of the delamination front when the arm of the DCB was subjected to a displacement of $9.4 \mathrm{~mm}$ (in accordance with the experimental measurements). For comparison, figure 8 shows the experimentally determined strain distribution derived from surface strain measurements measured using a strain gauge located at $34 \mathrm{~mm}$; as the delamination grew passed the strain gauge, the longitudinal strain distribution associated with the delamination could be determined.

Comparing figures 7 and 8 , it can be seen that they share similar features (note that the delaminated portion of the DCB specimen in both figures is in the positive $\mathrm{x}$ direction, with the delamination front located at the origin). Approaching the delamination front from the intact part of the $\mathrm{DCB}$, there is a rapid increase in the surface strain from about $15 \mathrm{~mm}$ ahead of the delamination front (i.e. from about $\mathrm{x}=$ $15 \mathrm{~mm}$ in the figures). The peak compressive surface strain is reached about $2 \mathrm{~mm}$ (model) and $4 \mathrm{~mm}$ (experiment) beyond the delamination front position, i.e. into the delaminated portion of the DCB. After the peak compressive strain (which corresponds to the smallest radius of curvature of the cantilever arms), the strain reduces linearly for both the FE model and experiment, although the rate of reduction of the compressive strains is lower for the experimental results.

The differences between the FE model of the surface strains and the experimental measurements are due to the fibre bridging. Improvements to the $\mathrm{FE}$ model could be made, but there is no way of incorporating fibre bridging into the FE model without using the experimental data to develop a semi-empirical FE model incorporating the bridging forces (see e.g. [12]). In the current work, an alternative approach is available, i.e. using the measured strain distribution from the surfacebonded strain gauges, in order to be able to predict the reflected spectra. The optical 
parameters used in the prediction using OptiGrating were uniform apodization, a Poisson's ratio for the optical fibre of 0.16 and photo-elastic coefficients $p_{11}=0.113$ and $p_{12}=0.252$, with an index modulation, $\Delta n$, of 0.000219 [7].

Figure 9(a) shows the predicted spectrum for a delamination length of $34 \mathrm{~mm}$, where the prediction is based on the surface strains measured by the strain gauges. Figures 9(b) shows an experimentally-recorded reflected spectrum for a delamination length of $34 \pm 1 \mathrm{~mm}$ (the uncertainty relates to the precise location of the delamination front obtained from the photographs). It can be seen that there is good agreement between the predicted and the experimentally determined reflected spectra, and this agreement has been used to derive the delamination lengths for all of the spectra recorded in the tests. Within the predicted spectrum of figure 9(a), the feature in the perturbation which corresponds to the position of the delamination front is the minimum in the perturbation. Consequently, it has been assumed that, for each experimentally measured spectrum, the delamination front position corresponds to the location of the minimum in the perturbation. The delamination length corresponding to the perturbation in each spectrum has then been calculated using the wavelength difference between the low-wavelength end of the spectrum and the position of the minimum in the perturbation, as a fraction of the full-width at half-maximum of the spectrum. This fraction is then converted to a length, assuming linearity between the spectral bandwidth and the physical length of the sensor $(60 \mathrm{~mm})$.

Figure 10 shows the CFBG sensor measurements of delamination length plotted against the photographically determined delamination length for three DCB specimens. The results show that the delamination lengths measured using the CFBG are within about $4 \mathrm{~mm}$ of the photographically measured delamination lengths, over the entire 60 mm length of the CFBG sensor. However, it should be pointed out that the accuracy 
with which the delamination length can be measured will be a function of the material and geometry of the structure. For example, for the present geometry, thicker DCB arms will reduce the precision of the measurements.

\section{Concluding remarks}

Surface-bonded CFBG sensors have been used to monitor the growth of delaminations in DCB specimens fabricated from transparent, unidirectionally reinforced glass fibre/epoxy resin. A perturbation in the CFBG reflected spectra, consisting of a dip in the reflected intensity, indicated the presence of the delamination front, since the perturbation was associated with high surface strain gradients in the vicinity of the delamination front. Significant fibre bridging was associated with the delamination as is common in these tests. In order to predict the reflected CFBG spectrum, surface strain measurements obtained independently using surface-mounted strain gauges have been used, with the predictions being in good agreement with experiment. Comparison between the photographically determined delamination lengths and the CFBG results showed that the position of the growing delamination can be determined to within about $4 \mathrm{~mm}$. Implementation of this technique for real applications will normally benefit from a finite-element model of the expected strain distribution in the presence of a delamination/disbond in order to validate the interpretation of the reflected spectra. 


\section{References}

[1] Wei Z, Yam LH, Cheng L. Detection of internal delamination in multi-layer composites using wavelet packets combined with modal parameter analysis. Composite Structures 2004;64:377-387.

[2] Todoroki A, Tanaka Y. Delamination identification of cross-ply graphite/epoxy composite beams using electric resistance change method. Composites Science and Technology 2002;62:629-639.

[3] In press: Amenabar I, Mendikute A, López-Arraiza A, Lizaranzu M, Aurrekoetxea J. Comparison and analysis of non-destructive testing techniques suitable for delamination inspection in wind turbine blades. Composites: Part B 2011.

[4] Raišutis R, Jasiūnienė E, Šliteris R, Vladišauskas A. The review of nondestructive testing techniques suitable for inspection of the wind turbine blades. ULTRAGARSAS Journal 2008;63;26-30.

[5] Gebremichael Y M, Li W, Boyle W J O, Meggitt B T, Grattan K T V, McKinley B, Fernando G F, Kister G, Winter D, Canning L, Luke S. Integration and assessment of fibre Bragg grating sensors in an all-fibre reinforced polymer composite road bridge. Sensors and Actuators 2005; A118, 78-85.

[6] Palaniappan J, Ogin SL, Thorne AM, Reed GT, Crocombe AD, Capell TF, Tjin SC, Mohanty L. Disbond growth detection in composite-composite single-lap joints using chirped FBG sensors. Composites Science and Technology 2008;68:2410-2417.

[7] Palaniappan J, Wang H, Ogin SL, Thorne A, Reed GT, Tjin SC, McCartney LN. Prediction of the reflected spectra from chirped fibre Bragg gratings embedded 
within cracked cross-ply laminates. Measurement Science and Technology 2006;17:1609-1614.

[8] Takeda S, Okabe Y, Takeda N. Monitoring of Delamination Growth in CFRP Laminates using Chirped FBG Sensors. Journal of Intelligent Material Systems and Structures 2008;19:437-444.

[9] Palaniappan J. 2008. The use of a CFBG sensor for detecting damage in composite laminates and adhesively bonded joints. Ph.D. University of Surrey.

[10] Smart Fibres Ltd. W4 Laser Interrogator: User guide and Smartsoft interface instructions.

[11] Lee J-J, Ogin SL, Smith PA "Interlaminar fracture toughness of glass fibre laminates additionally reinforced with carbon beads", Fifth Symposium on Composite Materials: Fatigue and Fracture, ASTM, Atlanta, Georgia, May 1993; published as ASTM STP 1230, R H Martin, Ed, pp38-60, 1995

[12] Stutz S, Cugnoni J, Botsis J. Studies of mode I delamination in monotonic and fatigue loading using FBG wavelength multiplexing and numerical analysis. Composites Science and Technology 2011;71;443-449.

[13] OptiGrating, Integrated and Fibre Optical Gratings Design Software (version 4.2), Optiwave Corporation, Canada.

\section{Figure Legends}

Figure 1 - (a) Schematic diagram showing plan (above) and edge (below) views of a specimen; (b) images of a specimen, showing the same views.

Figure 2 - $\quad$ A photographic comparison of a specimen with different delamination lengths (note: the delamination length indicated has developed from the end of the $40 \mathrm{~mm}$ long insert). 
Figure 3 - $\quad$ A typical plot of toughness against delamination length for the DCB specimens tested.

Figure 4 - $\quad$ Fibre bridging developing between the arms of the unidirectionally-reinforced DCB specimen; the approximate position of the delamination front is indicated (the red marks on the specimen indicate the delamination lengths at which photographs were taken)

Figure 5 - $\quad$ A typical plot of strain measured by strain gauges SG1 to SG6 against delamination length.

Figure 6 - The reflected spectra from a specimen with delamination lengths of (a) 0 mm and $20 \mathrm{~mm}$; (b) $20 \mathrm{~mm}$ and $39.5 \mathrm{~mm}$; (c) $39 \mathrm{~mm}$ and $57.1 \mathrm{~mm}$.

Figure 7 - $\quad$ Strain distribution extracted from FEA modelling, for a delamination that is 34 mm in length.

Figure 8 - $\quad$ Strain distribution associated with a delamination $34 \mathrm{~mm}$ long (derived from the surface strains measured by a strain gauge located $34 \mathrm{~mm}$ from the nylon insert).

Figure 9 - $\quad$ (a) Predicted spectrum for a delamination with a length of $34 \mathrm{~mm}$;

(b) experimentally-determined reflected spectrum for a delamination with a length of $34 \pm 1 \mathrm{~mm}$.

Figure 10 - A plot of the delamination lengths determined using the CFBG sensor plotted against the photographically recorded delamination lengths. 


\section{$\underline{\text { Figures }}$}

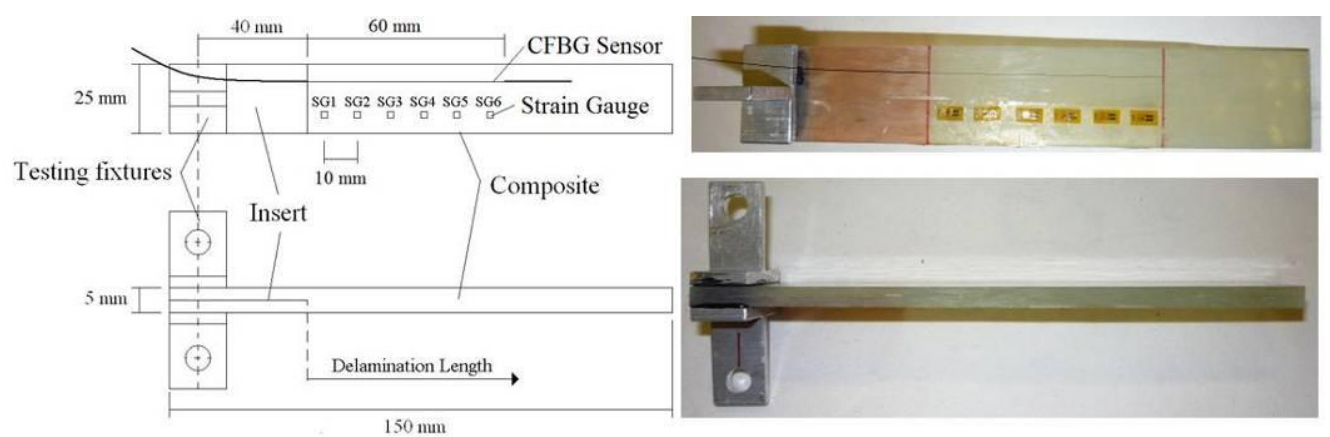

(a)

(b)

Figure 1 - (a) Schematic diagram showing plan (above) and edge (below) views of a specimen; (b) images of a specimen, showing the same views.

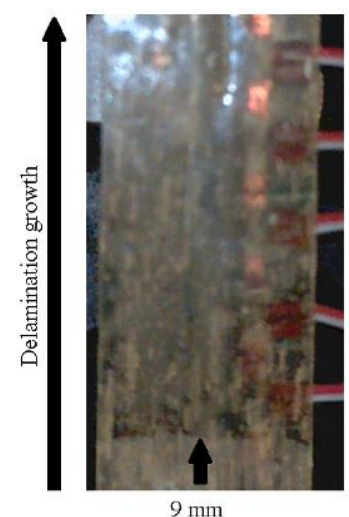

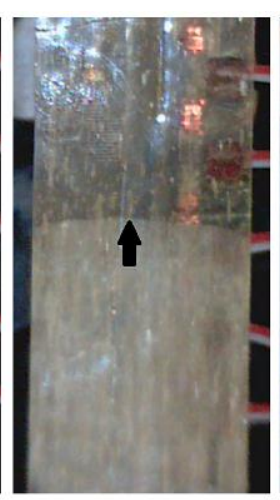

$34.5 \mathrm{~mm}$

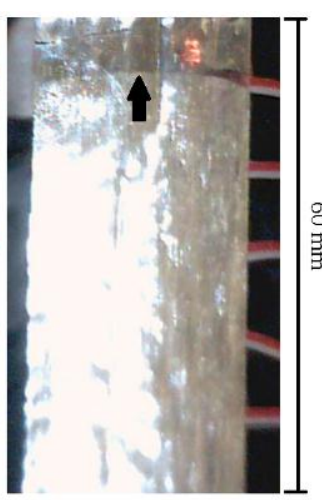

$51 \mathrm{~mm}$

Figure 2 - A photographic comparison of a specimen with different delamination lengths (note: the delamination length indicated has developed from the end of the $40 \mathrm{~mm}$ long insert).

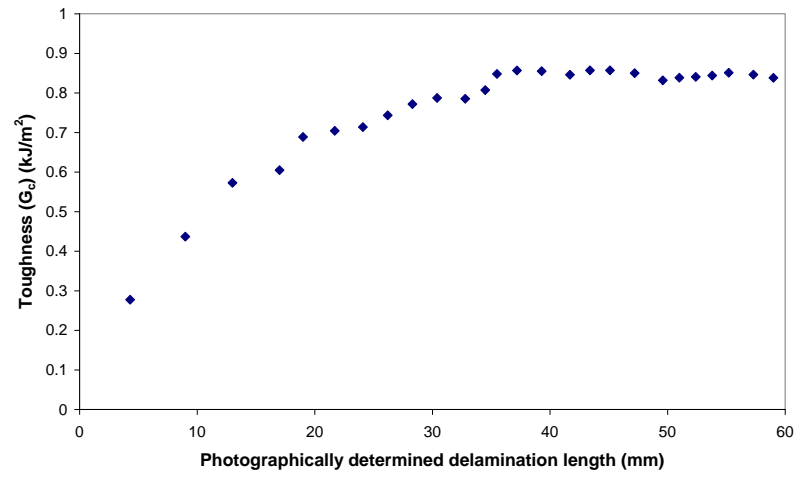

Figure 3 - A typical plot of toughness against delamination length for the DCB specimens tested. 


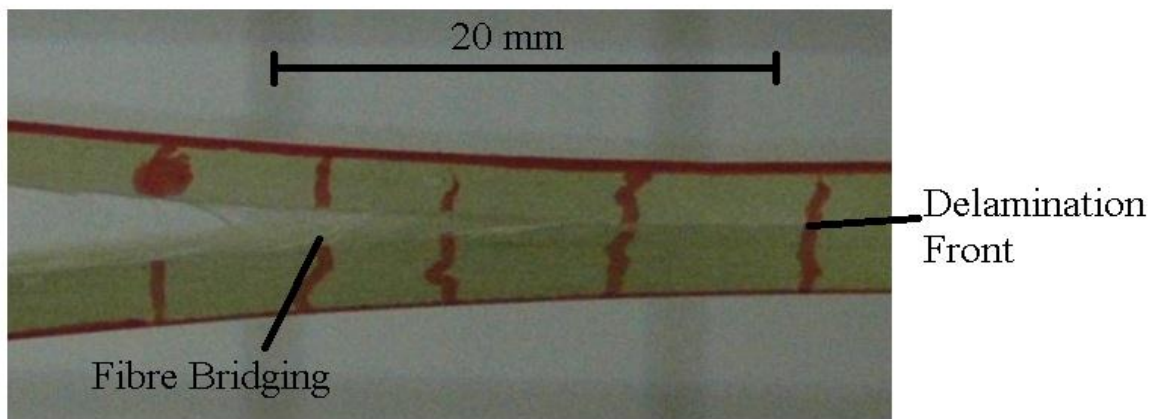

Figure 4 - Fibre bridging developing between the arms of the unidirectionally-reinforced DCB specimen; the approximate position of the delamination front is indicated (the red marks on the specimen indicate the delamination lengths at which photographs were taken)
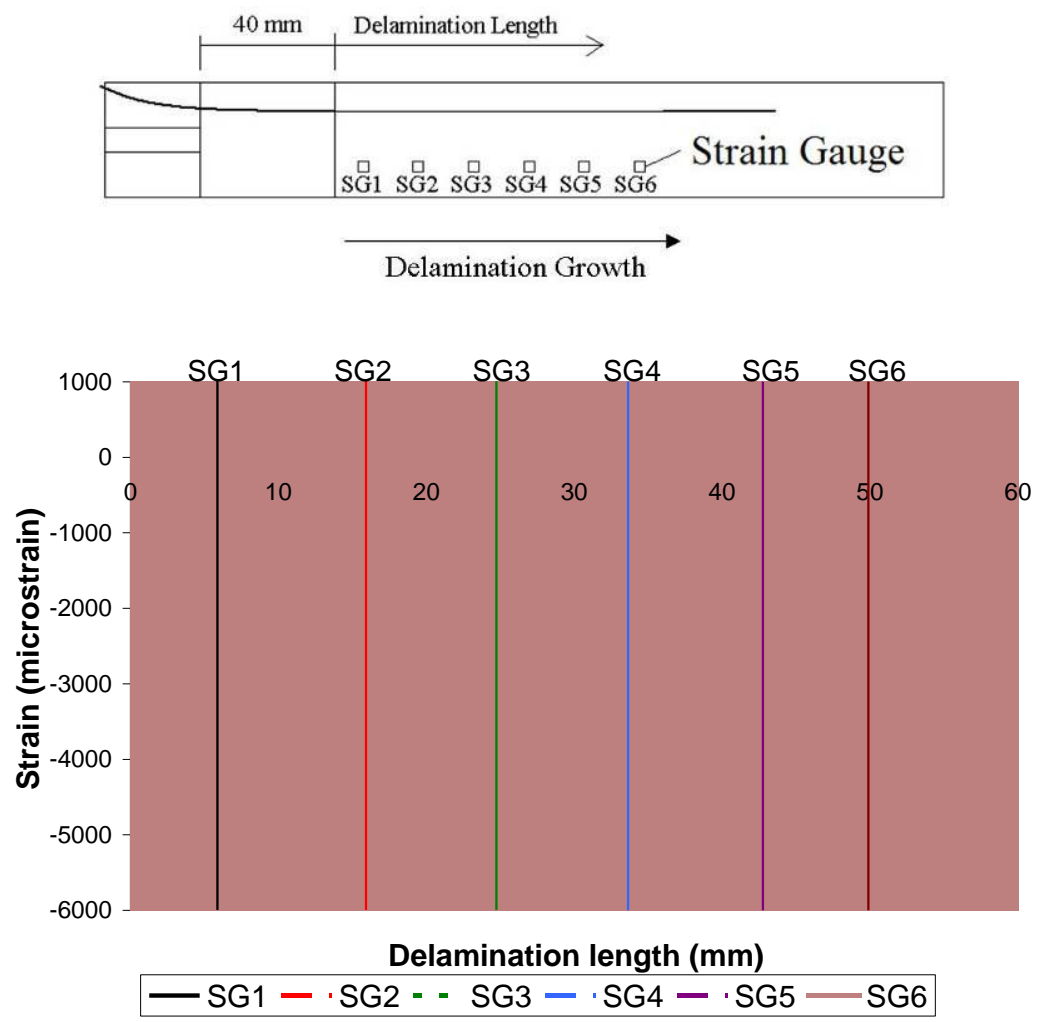

Figure 5 - A typical plot of strain measured by strain gauges SG1 to SG6 against delamination length. 


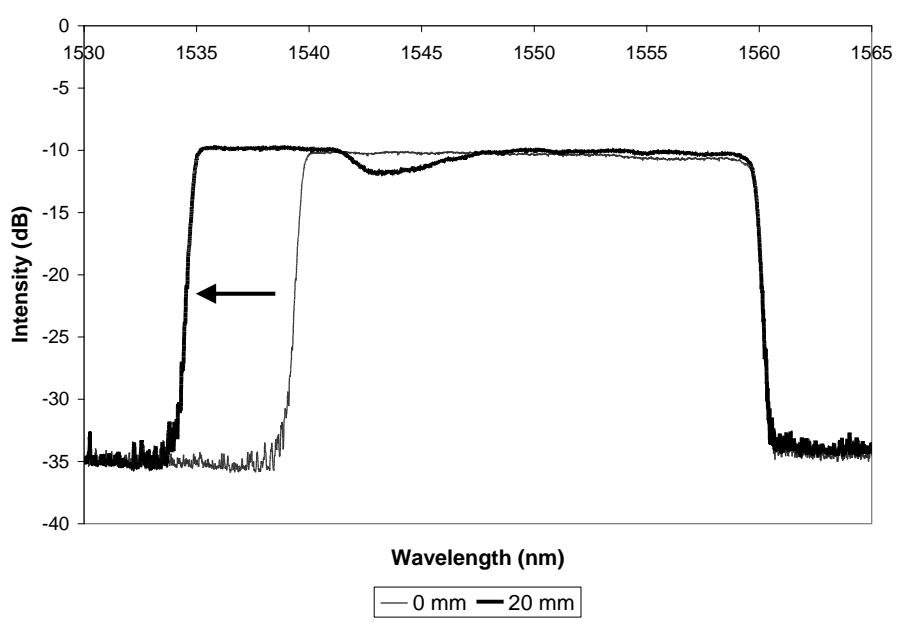

(a)

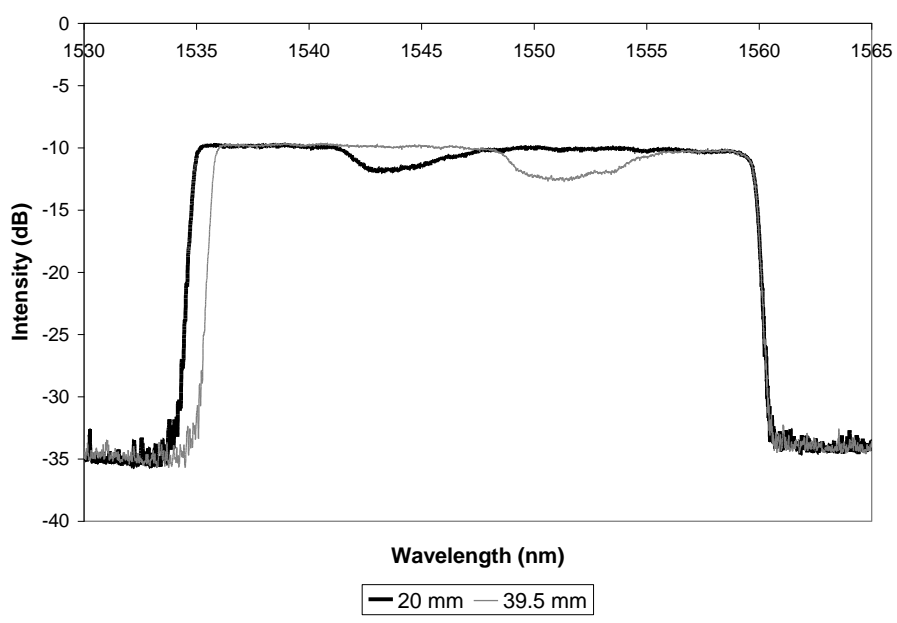

(b)

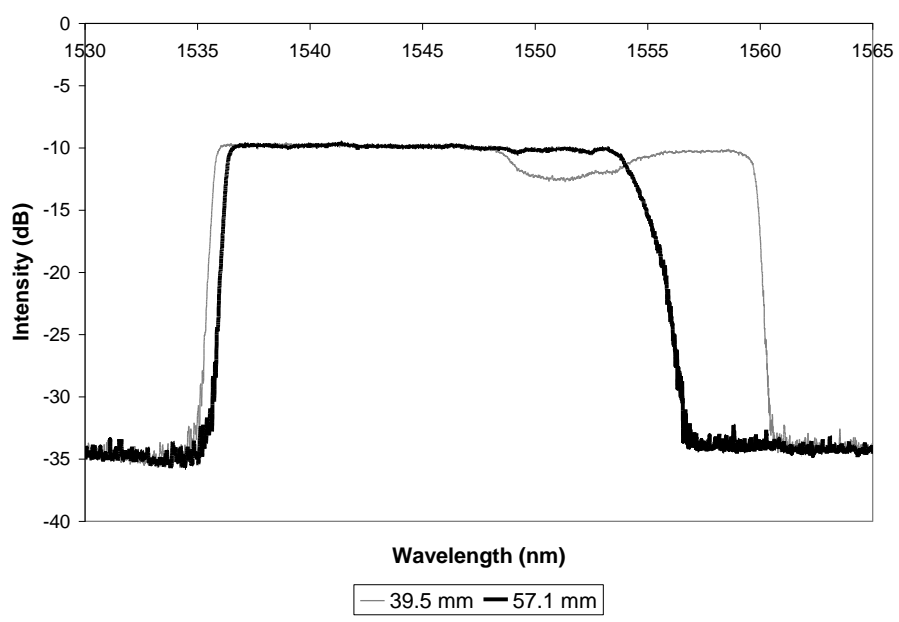

(c)

Figure 6 - The reflected spectra from a specimen with delamination lengths of (a) 0 mm and 20 mm; (b) $20 \mathrm{~mm}$ and $39.5 \mathrm{~mm}$; (c) $39 \mathrm{~mm}$ and $57.1 \mathrm{~mm}$. 


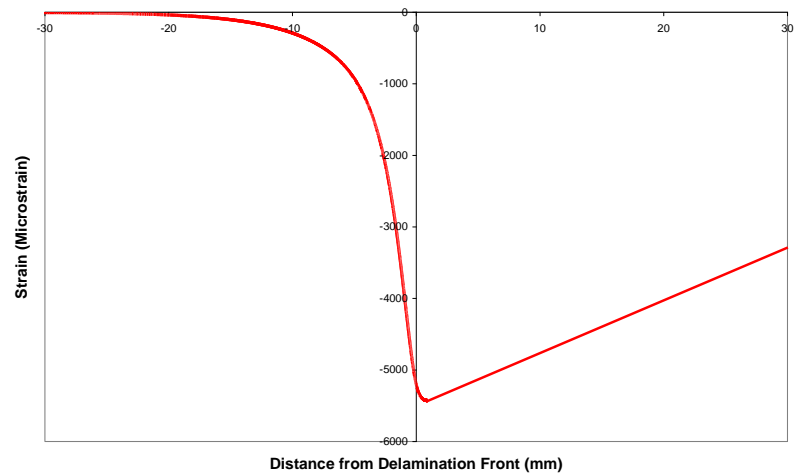

Figure 7 - Strain distribution extracted from FEA modelling, for a delamination that is $\mathbf{3 4} \mathbf{~ m m}$ in length.

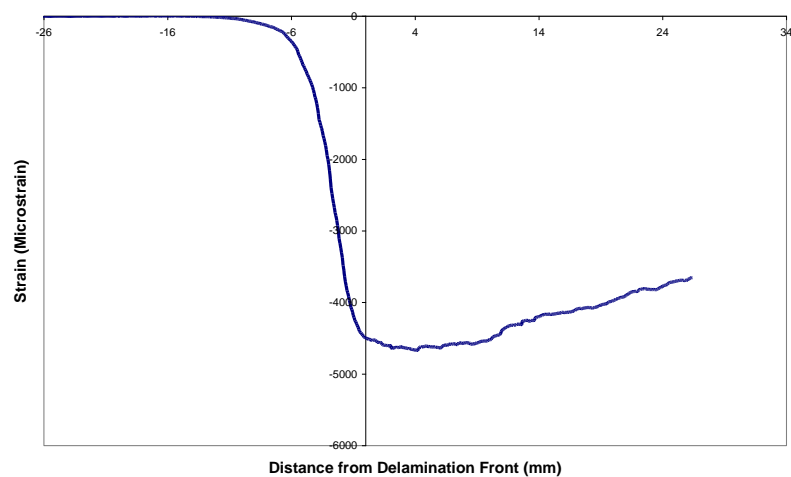

Figure 8 - Strain distribution associated with a delamination $34 \mathrm{~mm}$ long (derived from the surface strains measured by a strain gauge located $34 \mathrm{~mm}$ from the nylon insert)

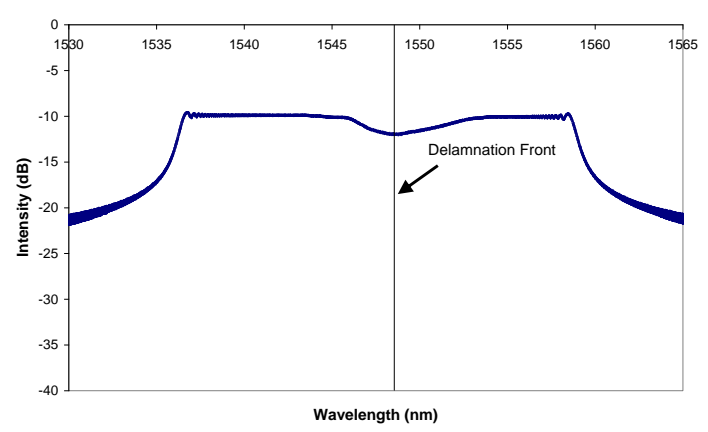

(a)

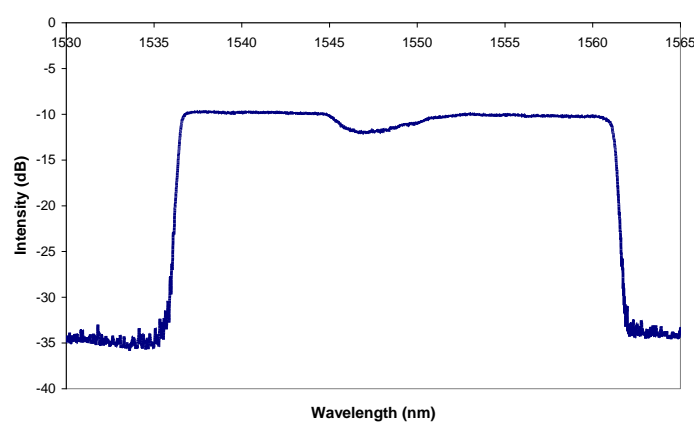

(b)

Figure 9 - (a) Predicted spectrum for a delamination with a length of $34 \mathrm{~mm}$; (b) experimentallydetermined reflected spectrum for a delamination with a length of $34 \pm 1 \mathrm{~mm}$. 


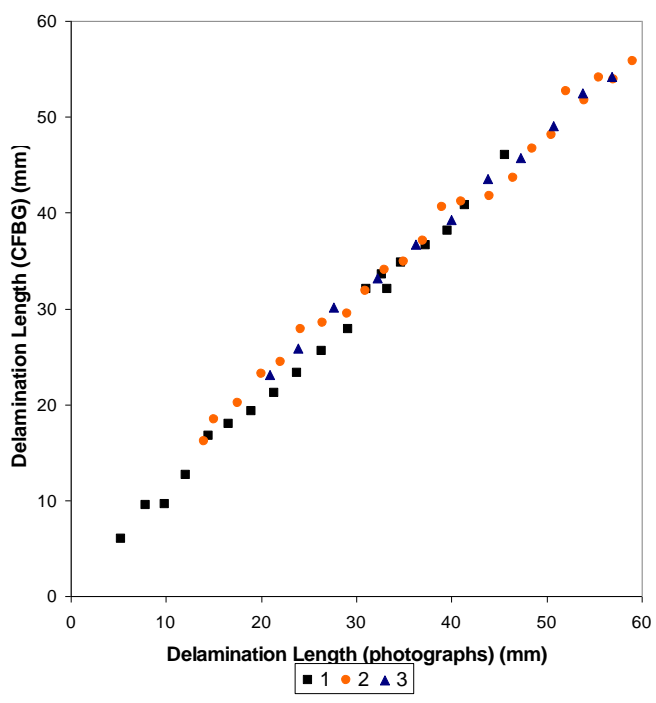

Figure 10 - A plot of the delamination lengths determined using the CFBG sensor plotted against the photographically recorded delamination lengths.

\section{Tables}

Table 1 - Composite material properties.

\begin{tabular}{|c|c|c|c|c|c|c|c|c|}
\hline $\mathrm{E}_{1}(\mathrm{GPa})$ & $\mathrm{E}_{2}(\mathrm{GPa})$ & $\mathrm{E}_{3}(\mathrm{GPa})$ & $\mathrm{G}_{12}(\mathrm{GPa})$ & $\mathrm{G}_{13}(\mathrm{GPa})$ & $\mathrm{G}_{23}(\mathrm{GPa})$ & $v_{12}$ & $v_{13}$ & $v_{23}$ \\
\hline 43 & 13 & 13 & 4 & 4 & 4.64 & 0.3 & 0.091 & 0.4 \\
\hline
\end{tabular}

